\title{
Heavy Quasiparticles in Yb Compounds: The Renormalized Band Approach
}

\author{
G. ZWICKNAGL*
}

Institut fuer Mathematische Physik, TU Braunschweig, Mendelssohnstr. 3, 38106 Braunschweig, Germany

\begin{abstract}
Results for the magnetic-field-induced changes of the heavy quasiparticles in $\mathrm{YbRh}_{2} \mathrm{Si}_{2}$ are presented. The bands are determined by means of the Renormalized Band method. The progressive de-renormalization of the quasiparticles in the magnetic field, as well as the many-body enhancement of the Zeeman splitting are accounted for, using field-dependent quasiparticle parameters deduced from Numerical Renormalization Group studies.
\end{abstract}

DOI: 10.12693 /APhysPolA.126.286

PACS: 71.10.+w, 71.10.Hf, 71.27.+a, 75.30.Mb

\section{Introduction}

The temperature versus magnetic field phase diagram of the heavy fermion (HF) compound $\mathrm{YbRh}_{2} \mathrm{Si}_{2}$ exhibits numerous anomalies $[1,2]$. The present paper focuses on the anomalies which are observed in various thermodynamic and transport properties in the Fermi liquid state in high magnetic fields [3].

\section{Model and methods}

The anomalous electronic properties of metals containing lanthanide atoms on regular lattice sites are conveniently described in terms of the f-electron Green's function

$$
\boldsymbol{G}_{f}(\boldsymbol{k} \omega)=\left(\boldsymbol{G}_{f ; l o c}^{-1}(\omega)-(\boldsymbol{W}(\boldsymbol{k} \omega)-\boldsymbol{w}(\omega))\right)^{-1},
$$

where $\boldsymbol{k}$ and $\omega$ denote wave vector and frequency, $\boldsymbol{G}_{f ; l o c}^{-1}$ is the fully renormalized local f-Green's function and $\boldsymbol{W}(\boldsymbol{k} \omega), \boldsymbol{w}(\omega)$ account for the hybridization with the conduction states. (For the notation see [4].) As written, the Green's functions are matrices in the basis of the local Crystalline Electric Field (CEF) eigenstates. The lowenergy excitations - the heavy fermions - are obtained by linearizing the frequency dependence of $\boldsymbol{G}_{f: l o c}^{-1}(\omega)$, which leads to effective band structure problems for the poles and residues of $\boldsymbol{G}_{f}(\mathbf{k} \omega)$. They are calculated by means of the Renormalized Band (RB) method, which combines material-specific ab initio methods and phenomenological considerations in the spirit of Landau. The central idea is to account for the local many-body effects contained in the expansion of $\boldsymbol{G}_{f ; l o c}^{-1}(\omega)$ by introducing a single parameter, which is subsequently fitted to the coefficient of the linear specific heat. The scheme has successfully described the Fermi liquid state of various HF systems [5-8]. Assuming that an external magnetic field affects the conduction states only weakly it is conveniently extended to the case of finite external magnetic fields $[9,10]$

$$
\begin{aligned}
& \boldsymbol{G}_{f}(\boldsymbol{k} \omega ; B)= \\
& \quad\left(\boldsymbol{G}_{f ; l o c}^{-1}(\omega ; B)-\boldsymbol{G}_{f ; l o c}^{-1}(\omega)+\boldsymbol{G}_{f}^{-1}(\boldsymbol{k} \omega)\right)^{-1} .
\end{aligned}
$$

\footnotetext{
*e-mail: g.zwicknagl@tu-bs.de
}

In the present calculation, the variation with magnetic field of the quasiparticle parameters is obtained from fits to field-dependent quasiparticle Density of States (DOS) of the single-impurity Anderson model [11, 12]. The resulting effective band structure is calculated with [13].

\section{Results}

The results for the DOS are displayed in Fig. 1. The strongly anisotropic hybridization between the $f$-states and the conduction bands leads to pronounced van Hovesingularities in the DOS which correspond to Lifshitz transitions of the iso-energy surfaces. The topologies of

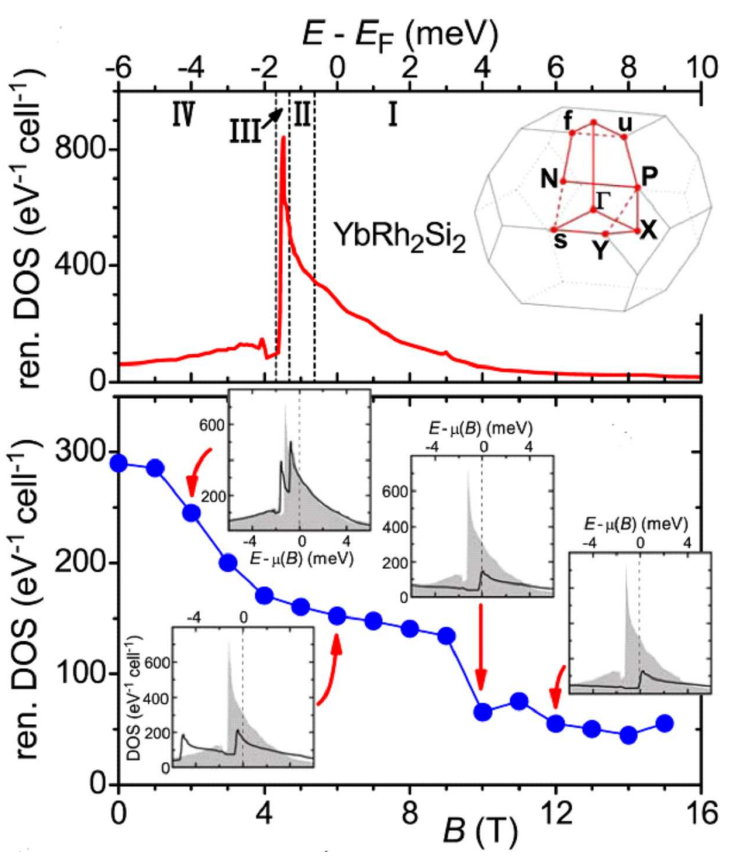

Fig. 1. Quasiparticle DOS of $\mathrm{YbRh}_{2} \mathrm{Si}_{2}$ at $B=0$ as calculated from the Renormalized Bands (upper panel) and variation with magnetic field in the basal plane of the quasiparticle DOS at the Fermi energy (lower panel). The DOS at different magnetic fields, displayed in the insets, clearly show a Zeeman splitting of the van Hove singularity. The inset in the upper panel displays the Brillouin zone of the tetragonal body-centered lattice and the special points. 
the latter define four different energy regimes, indicated by vertical lines in the upper panel. The existence of these different regimes is reflected in the variation with magnetic field of the quasiparticle DOS at the Fermi energy. The characteristic features are (i) a continuous decrease resulting from the de-renormalization of the heavy quasiparticles and (ii) discontinuous changes at well defined values of the magnetic field, resulting from Lifshitz transitions of the Fermi surface. The variation with magnetic field of the DOS at the Fermi energy agrees well with the measured DOS as can be seen from Fig. 2 .

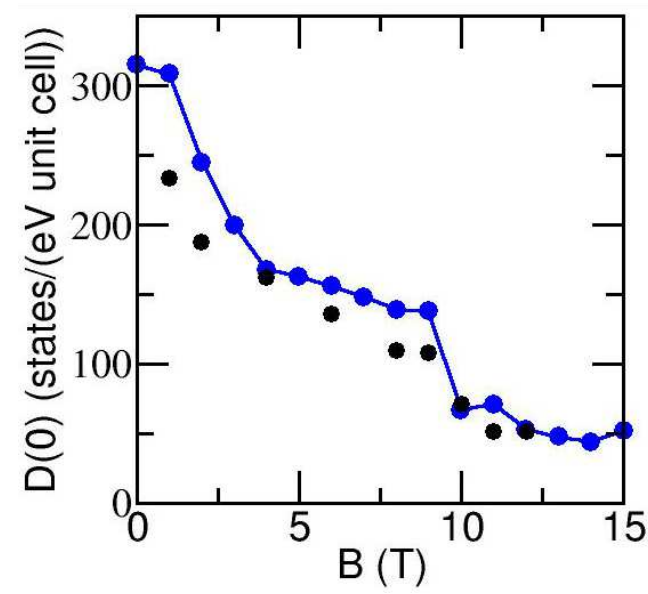

Fig. 2. Comparison with experiment: Variation with magnetic field in the basal plane of the quasiparticle DOS at the Fermi level $\mathrm{D}(0)$, derived from the RB calculation (blue circles), and measured DOS deduced from the specific heat coefficient measured at ambient pressure (black circles)[1].

\section{Conclusions}

In conclusion, the following picture emerges: To quantitatively understand the unusual low-temperature behavior of $\mathrm{YbRh}_{2} \mathrm{Si}_{2}$ in high magnetic fields we have to account for both local many-body effects and coherence structures from the periodicity of the lattice. The magnetic-field-induced topological transitions of the Fermi surface are also reflected in the transport properties of $\mathrm{YbRh}_{2} \mathrm{Si}_{2}[14,15]$. The results suggest that it will be difficult to extract the zero-field Fermi surface from de Haas - van Alphen or Shubnikov - de Haas experiments. In fact, it should be determined from AngleResolved Photoemission (ARPES) studies [16].

\section{Acknowledgments}

It is a pleasure to thank S. Friedemann, P. Gegenwart, C. Geibel, F. Steglich, S. Tornow, T. Westerkamp, S. Wirth for helpful discussions and F. B. Anders for making available the NRG code.

\section{References}

[1] P. Gegenwart, Y. Tokiwa, T. Westerkamp, F. Weickert, J. Custers, J. Ferstl, C. Krellner, C. Geibel, P. Kerschl P, K.-H. Müller, F. Steglich, New J. Phys. 8, 171 (2006).

[2] P. Gegenwart, Q. Si, F. Steglich, Nat. Phys. 4, 186 (2008).

[3] Y. Tokiwa, P. Gegenwart, T. Radu, J. Ferstl, G. Sparn, C. Geibel, F. Steglich, Phys. Rev. Lett. 94, 226402 (2005).

[4] P. Fulde, J. Keller, G. Zwicknagl, Solid State Physics 41, 1 (1988).

[5] G. Zwicknagl, Adv. Phys. 41, 203 (1992).

[6] G. Zwicknagl, Phys. Scr. T 49, 34 (1993).

[7] P. Fulde, P. Thalmeier, G. Zwicknagl, Solid State Physics 60, 1 (2006).

[8] S. Ernst, S. Kirchner, C. Krellner, C. Geibel, G. Zwicknagl, F. Steglich, S. Wirth, Nature 474, 362 (2011).

[9] G. Zwicknagl, J. Phys.: Condens. Matter 23, 094215 (2011).

[10] G. Zwicknagl, J. Phys. Soc. Jpn. 80, SA010 (2011).

[11] A.C. Hewson, A. Oguri, D. Meyer, Eur. Phys. J. B 40, 177 (2004); A.C. Hewson, J. Bauer, W. Koller, Phys. Rev. B 73, 045117 (2006); J. Bauer, A.C. Hewson, Phys. Rev. B 76, 035119 (2007).

[12] R. Peters, T. Pruschke, F.B. Anders, Phys. Rev. B 74, 245114 (2006).

[13] N.E. Christensen, Int. J. Quantum Chem. XXV, 233 (1984).

[14] H. Pfau, R. Daou, S. Lausberg, H.R. Naren, M. Brando, S. Friedemann, S. Wirth, T. Westerkamp, U. Stockert, P. Gegenwart, C. Krellner, C. Geibel, G. Zwicknagl, F. Steglich, Phys. Rev. Lett. 110, 256403 (2013).

[15] A. Pourret, G. Knebel, T.D. Matsuda, G. Lapertot, J. Flouquet, J. Phys. Soc. Jpn. 82, 053704 (2013).

[16] K. Kummer et al., (preprint). 